DE DE GRUYTER

OPEN

G

BULGARIAN ACADEMY OF SCIENCES

CYBERNETICS AND INFORMATION TECHNOLOGIES • Volume 15, No 5

Special Issue on Control in Transportation Systems

Sofia • 2015

Print ISSN: 1311-9702; Online ISSN: 1314-4081

DOI: 10.1515/cait-2015-0013

\title{
Macroscopic Traffic Flow Control via State Estimation
}

\author{
H. Abouaïssa ${ }^{1}$, H. Majid ${ }^{2}$ \\ ${ }^{I}$ Université Lille Nord France, F-59000 Lille, France. U-Artois, LGI2A, (EA. 3926) Technoparc \\ Futura, F-62400 Béthune, France \\ ${ }^{2}$ University of Sulaimani, Faculty of Engineering Sciences, Department of Civil Engineering, \\ Sulaimani, Iraq \\ Emails: hassane.abouaissa@univ-artois.fr herishmm@gmail.com
}

\begin{abstract}
The studies presented in this paper deal with traffic control in case of missing data and/or when the loop detectors are faulty. We show that the traffic state estimation plays an important role in traffic prediction and control. Two approaches are presented for the estimation of the main traffic variables (traffic density and mean speed). The state constructors obtained are then used for traffic flow control. Several numerical simulations show very promising results for both traffic state estimation and control.
\end{abstract}

Keywords: Macroscopic model, estimation, freeway traffic control, sliding mode, algebraic method, differential flatness.

\section{Introduction}

The freeway traffic control and the advent of the Intelligent Transportation Systems (ITS), which are able to provide continuous forecasting of the traffic and allow alleviating the daily congestion phenomena, are closely related to the ability to have the whole needed information about the traffic behaviour and then the traffic state evolution. This information is usually provided by a set of loop detectors or any other sensors, such as video camera and others, already installed along the freeway infrastructure. Nevertheless, like any other device, such sensors face several problems, such as failure, usury and default, as well as the high cost of installation and maintenance. In addition, the loop detectors could also undermine the road paved and accelerate the deterioration of the infrastructure. The state estimation and reconstruction of the missing data for a faulty detector play an important role for both traffic forecasting and control. 
The amount of attention devoted to the development of an estimation strategy using a traffic flow model has been relatively small compared to the modeling stage. Among these works, $\mathrm{Nahi}$ and Trive di [18] shows an interesting method for data processing and estimation of the traffic density closely in homogeneous situations. Notice that the most widely used tools for traffic state estimation are Kalman filters and their extensions. Thus, G a z i s and K n a p p [10], K n a p p [12] have proposed a Kalman filtering techniques for data processing. Such a method is based on time series of the mean speed and flow data from each detector and then it generates crude estimates of the vehicle counts. In [21] an extended Kalman filter method was utilized for traffic state estimation, using second order models. Kohan [13] has designed a first order sliding mode state estimator for the estimation of both speed and density. Although the proposed method is robust against bounded modeling errors and various disturbances, it suffers from chattering phenomena. Sun et al. [20] have derived a density estimator at unmonitored locations along a freeway, from the so-called Switching Mode Model (SMM). Sun et al. have proposed an estimator based on a Mixture Kalman Filter (MKF) algorithm using also the SMM. Mihaylova and Boel [17] have used a particle filter for density estimation/prediction. Most of these works have dealt with state estimation for traffic prediction and forecasting.

In this paper we propose a combination of the state estimation for traffic flow control purpose. Two approaches are provided. The first one aims at solving the problem of the chattering phenomena using high order sliding mode techniques. The second method is based on new setting of numerical differentiation. The obtained estimators are then used for freeway traffic flow control.

Our paper is organized as follows. Section 2 recalls the used macroscopic model. The sliding mode and algebraic methods are presented in Section 3 and then applied to the state estimation. Section 4 details the developed traffic flow control algorithm using the state estimation. Numerical simulations and results are provided in Section 5. Finally, the last section summarizes some conclusions and further works.

\section{A macroscopic traffic flow model}

\subsection{A modified second order continuum model}

As an illustration consider the following simple freeway stretch divided into three segments.

The studied system is governed by the following space discretized equation, called a conservation law

$$
\dot{\rho}_{i}(t)=\frac{1}{L_{i} \lambda_{i}}\left[q_{i-1}(t)-q_{i}(t)+a_{i} r_{i}(t)-b_{i} p_{i}(t)\right]
$$

where: $\dot{\rho}_{i}(t)$ in (veh/km per lane) is the traffic density in segment $I ; q_{i}(t)$ denotes the traffic volume in (veh/h); $L_{i}$ and $\lambda_{i}$, are the segment length and the number of 
lanes in segment $i$, respectively; $a_{i}$ and $b_{i}$ are binary variables which indicate respectively the presence or the absence of an on-ramp $r_{i}(t)$ and an off-ramp $p_{i}(t)$.

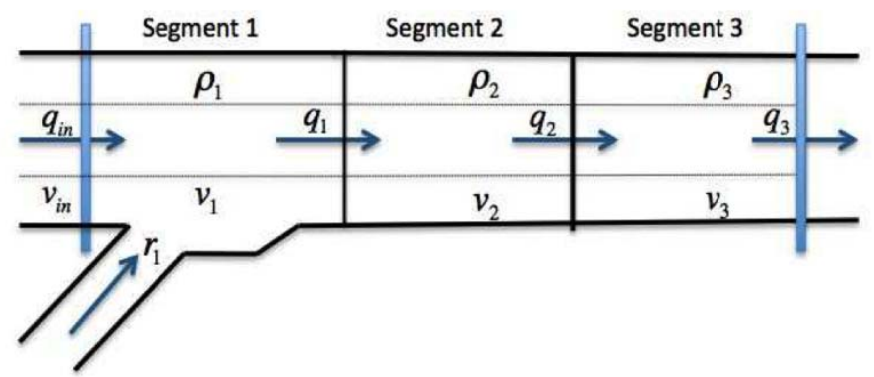

Fig. 1. Example of freeway stretch with three segments

The traffic volume $q_{i}(t)$ is related to the traffic density and the mean speed $v_{i}(t)$ thanks to the following expression borrowed by Hillige s and Weidlich [11] (see also [13]):

$$
q_{i}(t)=a v_{i+1}(t) \rho_{i}(t)+(1-\alpha) \rho_{i+1}(t) v_{i+2}(t)
$$

for $i=1, \ldots, N-1$ and $q_{N}(t)=\rho_{N}(t) v_{N}(t)$, where $0 \leq \alpha \leq 0$ represents an appropriate weighting factor, which generally needs to be identified. Since the number of vehicles leaving section $i$ is mainly defined as a function of segment states rather than the traffic conditions further downstream, this parameter is assumed to be close to 1 .

The mean speed is defined thanks to the following differential equation

$$
\dot{v}_{i}(t)=\frac{1}{\tau}\left[V_{e}\left(\rho_{i}(t)\right)-v_{i}(t)\right]+\frac{\xi}{L_{i}} v_{i}(t)\left[v_{i-1}(t)-v_{i}(t)\right]-\frac{v}{\tau L_{i}}\left[\rho_{i+1}(t)-\rho_{i}(t)\right],
$$

where $V_{e}\left(\rho_{i}(t)\right)=v_{f, i} \exp \left[-\frac{1}{a}\left(\frac{\rho_{i}}{\rho_{c, i}}\right)^{a}\right]$ is the so-called "fundamental diagram" (see, e.g., [15]); $v_{f, i}$ is the free-flow speed, $a$ is a model parameter and $\rho_{c, i}$ represents the critical density of the segment $i$.

\subsection{Initial conditions}

From the example in Fig. 1 we assume that the traffic volume and the mean speed $\left(q_{\text {in }}(t), v_{\text {in }}(t)\right.$ ) are usually measured from the loop detector located at the uppermost boundary of the freeway section. We assume also stationary conditions at the exit segment. Then, for the last downstream segment $N, \rho_{N}(t)=\rho_{N+1}(t)$.

We consider then the following initial conditions:

- $q_{0}=q_{\mathrm{in}}, v_{0}=v_{\mathrm{in}}$,

- $\rho_{0}=\frac{q_{0}}{v_{0}}, v_{0} \neq 0$,

- $\rho_{N}=\rho_{N+1}$. 


\section{Nonlinear traffic state estimation}

The main objective of the traffic state estimation is to design an observer for a freeway stretch described by Equations (1), (2) and (3).

For a freeway stretch divided into $N$ segments, the set of state variables that govern the studied system are: $\left[\rho_{1} v_{1}, \ldots, \rho_{N} v_{N}\right] \in R^{2 N}$. We can easily observe that the system consists of $2 N$ equations with $2 N$ segment variables. Assume, following [21], that a set of traffic measurement devices, such as loop detectors, video cameras, etc., are installed along the freeway stretch at a separation of several kilometers, i.e., at the end of every $m$ segment and at on-ramps. Such devices provide measurements of the traffic flow, space mean speed and occupancy at every $T$ seconds. (For simulation purpose the occupancy rate in \% is converted into traffic density.) The measurements provided by the sensors allow the designed reconstructor to estimate the main traffic state variables for each segment. Having the freeway stretch with $N$ segments and $m$ sensors, the freeway system can be subdivided into approximately $M=N / m$ subsystems. Such systems subdivision is achieved in such a way that the subsystem $l+1$ has little influence on the dynamics of the mainstream subsystem $l, l=1,2, \ldots, M-1$ [13]. To reach this requirement, the length of the last segment of the sub- system $l$ must be small compared to the first segment of the downstream subsystem $l+1$. This condition means that the effect of the anticipation term in expression (3) for the last segment of the subsystem $l$ is negligible and one can consider the decentralized problem of the state estimation for each subsystem at a time.

\subsection{A sliding mode observer for traffic state estimation}

The sliding mode technique is related to the Variable Structure Systems (VSS) theory. It is essentially based on the resolution of differential equations with discontinuous right hand side, which was introduced by [4]. Historically, the twisting mode algorithm is the first 2nd order sliding mode algorithm known [5]. It features twisting around the origin of the 2nd order-sliding plane. The trajectories perform an infinite number of rotations while converging in a finite time to the origin $[9,19]$.

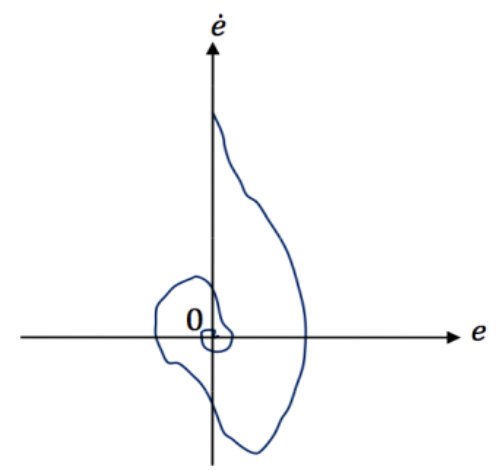

Fig. 2. Super-twisting algorithm phase trajectory where $e$ represents the error 
In the super-twisting algorithm, the trajectories of the 2nd order-sliding plane are also characterized by twisting around the origin (Fig. 2; e.g., [14] for a short review on the Super-twisting sliding mode observer and its application to traffic flow estimation).

Fig. 3 illustrates the structure of a super-twisting observer algorithm.

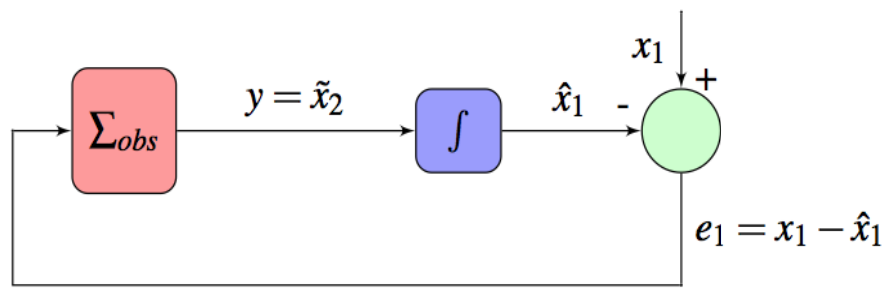

Fig. 3. Structure of the super-twisting observer

In the following lines we consider the example of Fig. 1 to show the design of the state estimation algorithm. In this example we assume that the output vector is $F=\left[F_{1} F_{2}\right]^{\mathrm{T}}=\left[\rho_{3} v_{3}\right]^{\mathrm{T}}$.

In terms of macroscopic modeling the studied freeway stretch of Fig. 1 is described as follows:

$$
\begin{gathered}
\left\{\begin{array}{l}
\dot{\rho}_{1}=\frac{1}{L_{1}}\left[q_{\text {in }}-\alpha \rho_{1} v_{2}-(1-\alpha) \rho_{2} v_{3}+r_{1}\right], \\
\dot{\rho}_{2}=\frac{1}{L_{2}}\left[\alpha \rho_{1} v_{2}+(1-2 \alpha) \rho_{2} v_{3}-(1-\alpha) \rho_{3} v_{3}\right], \\
\dot{\rho}_{3}=\frac{1}{L_{3}}\left[\alpha \rho_{2} v_{3}+(1-\alpha) \rho_{3} v_{3}-q_{\text {out }}\right]
\end{array}\right. \\
\left\{\begin{array}{l}
\dot{v}_{1}=\frac{1}{\tau}\left[V_{e}\left(\rho_{1}\right)-v_{1}\right]+\frac{\xi}{L_{1}} v_{1}\left[v_{i n}-v_{1}\right]-\frac{v}{L_{1}}\left[\rho_{2}(t)-\rho_{1}(t)\right]-\frac{\delta}{L_{1}} r_{1} v_{1}, \\
\dot{v}_{2}=\frac{1}{\tau}\left[V_{e}\left(\rho_{2}\right)-v_{2}\right]+\frac{\xi}{L_{2}} v_{2}\left[v_{1}-v_{2}\right]-\frac{v}{L_{2}}\left[\rho_{3}(t)-\rho_{2}(t)\right], \\
\dot{v}_{3}=\frac{1}{\tau}\left[V_{e}\left(\rho_{3}\right)-v_{3}\right]+\frac{\xi}{L_{3}} v_{3}\left[v_{2}-v_{3}\right]-\frac{v}{L_{3}}\left[\rho_{\text {out }}(t)-\rho_{3}(t)\right]=\dot{F}_{v} .
\end{array}\right.
\end{gathered}
$$

As above mentioned, in expressions (4) and (5), $\rho_{3}$ and $v_{3}$, are assumed to be known. It should be underlined that $q_{\text {out }}=\rho_{\text {out }} v_{\text {out }}$ forms the output measurements variables. If $\rho_{\text {out }}<\rho_{c}$, then $\rho_{\text {out }}=\rho_{3}$, else $\rho_{\text {out }}=\rho_{c}$. In addition, $v_{1}, v_{2}, v_{3} \leq v_{f}$.

The main objective of the observer is to estimate the traffic variables: $\rho_{1}, v_{1}$, $\rho_{2}, v_{2}$. Consider then that $\hat{\rho}$ and $\hat{v}$ are the estimated values of $\rho$ and $v$, respectively. Then, the observer errors can be defined by $e_{\rho}=\rho-\hat{\rho}$, and $e_{v}=v-\hat{v}$. Thus the proposed super-twisting observer can be expressed as: 
(6)

$$
\begin{aligned}
& \left\{\begin{array}{l}
\dot{\hat{\rho}}_{1}=\frac{1}{L_{1}}\left[q_{\text {in }}-\alpha \hat{\rho}_{1} \hat{v}_{2}-(1-\alpha) \hat{\rho}_{2} v_{3}+r_{1}\right], \\
\dot{\hat{\rho}}_{2}=\frac{1}{L_{2}}\left[\alpha \tilde{\rho}_{1} \hat{v}_{2}+(1-\alpha) \hat{\rho}_{2} v_{3}-(1-\alpha) \rho_{3} v_{3}\right]+z_{\hat{\rho} 2}, \\
\tilde{\rho}_{1}=\pi_{\rho} \operatorname{sig} n\left(\tilde{\rho}_{2}-\hat{\rho}_{2}\right), \\
z_{\hat{\rho} 2}=\beta_{2}\left|\tilde{\rho}_{2}-\hat{\rho}_{2}\right|^{\frac{1}{2}} \operatorname{sign}\left(\tilde{\rho}_{2}-\hat{\rho}_{2}\right),
\end{array}\right. \\
& \left\{\begin{array}{l}
\dot{\hat{\rho}}_{3}=\frac{1}{L_{3}}\left[\alpha \hat{\rho}_{2} v_{3}-(1-\alpha) \hat{\rho}_{3} v_{3}-q_{\text {out }}\right]+z_{\hat{\rho} 3}, \\
\tilde{\rho}_{2}=\pi_{\rho} \operatorname{sign}\left(\tilde{\rho}_{3}-\hat{\rho}_{3}\right), \\
z_{\hat{\rho} 3}=\beta_{3}\left|\tilde{\rho}_{3}-\hat{\rho}_{3}\right|^{\frac{1}{2}} \operatorname{sign}\left(\tilde{\rho}_{3}-\hat{\rho}_{3}\right) .
\end{array}\right.
\end{aligned}
$$

Analogically, the mean speeds estimation are obtained via the following observer:

$$
\left\{\begin{array}{l}
\dot{\hat{v}}_{1}=\frac{1}{\tau}\left[V_{\mathrm{e}}\left(\hat{\rho}_{1}\right)-\hat{v}_{1}\right]+\frac{\xi}{L_{1}} \hat{v}_{1}\left[v_{\mathrm{in}}-\hat{v}_{1}\right]-\frac{v}{L_{1}}\left[\hat{\rho}_{2}(t)-\hat{\rho}_{1}(t)\right]-\frac{\delta}{L_{1}} r_{1} \hat{v}_{1}, \\
\dot{\hat{v}}_{2}=\frac{1}{\tau}\left[V_{\mathrm{e}}\left(\hat{\rho}_{2}\right)-\hat{v}_{2}\right]+\frac{\xi}{L_{2}} \hat{v}_{2}\left[\tilde{v}_{1}-\hat{v}_{2}\right]-\frac{v}{L_{2}}\left[\hat{\rho}_{3}(t)-\hat{\rho}_{2}(t)\right]+z_{v 2}, \\
\tilde{v}_{1}=\pi_{v} \operatorname{sign}\left(\tilde{v}_{2}-\hat{v}_{2}\right), \\
z_{v 2}=\beta_{2}\left|\tilde{v}_{2}-\hat{v}_{2}\right| \frac{1}{2} \operatorname{sign}\left(\tilde{v}_{2}-\hat{v}_{2}\right) .
\end{array}\right.
$$

The output injections are of the form:

$$
\left\{\begin{array}{l}
\dot{\hat{v}}_{3}=\frac{1}{\tau}\left[V_{\mathrm{e}}\left(\hat{\rho}_{3}\right)-\hat{v}_{3}\right]+\frac{\xi}{L_{3}} \hat{v}_{3}\left[\tilde{v}_{2}-\hat{v}_{3}\right]-\frac{v}{L_{3}}\left[\rho_{\text {out }}(t)-\hat{\rho}_{3}(t)\right]+z_{v 3}, \\
\tilde{v}_{2}=\pi_{v} \sin g\left(\tilde{v}_{2}-\hat{v}_{2}\right), \\
z_{\rho 3}=\beta\left|v_{3}-\hat{v}_{3}\right|^{\frac{1}{2}} \sin g\left(\tilde{v}_{3}-\hat{v}_{3}\right) .
\end{array}\right.
$$

\subsection{Numerical differentiation for traffic state estimation}

Numerical differentiation is a very important but difficult ill-posed theoretical problem [16]. This algebraic technique starting in [5, 6], (see also, e.g., [7, 8]), provides a powerful tool for the estimation of the derivatives of a noisy signal. Its applications to traffic flow state estimation and control are still in their preliminary phase. In this section we will show how the use of such a method provides a very interesting alternative to the existing methods for state estimation, using the second order macroscopic model described in Section 2. The principle of proposed numerical differentiation was already described in [1].

Without loss of generality, consider the freeway stretch depicted in Fig. 1. The efficient design of the proposed estimator is made possible thanks to the 
observability definition given by $\mathrm{D}$ i o p and F li e s s $[2,3]$.

Definition. A nonlinear input-output is observable if, and only if, any system variable, a state variable for instance, is a differential function of the control and output variables, i.e., a function of those variables and their derivatives up to some finite order. We will say more generally that an unknown quantity may be determined if, and only if, it is expressible as a differential function of the control and output variables.

From this definition the state variables in the freeway stretch of Fig. 1, are defined as $S=\left[\rho_{1}, v_{1}, \rho_{2}, v_{2}, \rho_{3}, v_{3}\right]^{\mathrm{T}} \in R^{6}$. For the sake of simplicity, we assume that all segments are with the same length: $L=L_{i}, i=1,2,3$.

Let $F_{1}=\rho_{3}$ be the measured variable output. The remaining variables $\rho_{1}$ and $\rho_{2}$ can be expressed in term of $F_{1}$ and its time-derivatives up to two $\dot{F}_{1}$ and $\ddot{F}_{1}$ :

$$
\rho_{2}=\frac{L}{\alpha v_{3}} \dot{F}_{1}-\frac{(1-\alpha)}{\alpha} F_{1}+\frac{1}{\alpha v_{3}} q_{\text {out }},
$$

$$
\rho_{1}=\frac{L^{2}}{\alpha^{2} v_{2} v_{3}} \ddot{F}_{1}+\frac{L(3 \alpha-2)}{\alpha^{2} v_{2}} \dot{F}_{1}+\frac{v_{3}(1-\alpha)^{2}}{\alpha^{2} v_{2}} F_{1}+\frac{L}{\alpha^{2} v_{2} v_{3}} \dot{q}_{\text {out }}-\frac{1-2 \alpha}{\alpha^{2} v_{2}} q_{\text {out }} \text {. }
$$

If we consider that $F_{2}=v_{3}$ is measured, then $v_{1}$ and $v_{2}$ can be expressed as follows:

$$
\begin{gathered}
v_{2}=\frac{L}{\xi} \frac{\ddot{F_{2}}}{F_{2}}-\frac{L}{\xi \tau} \frac{V_{\mathrm{e} 3}}{F_{2}}+F_{2}+\frac{L}{\xi \tau}, \\
v_{1}=F_{2}+\frac{2 L}{\xi \tau}-\frac{L}{\xi} \frac{V_{\mathrm{e} 2}}{\sigma_{1}}+\frac{L}{\xi} \frac{\dot{F}_{2}}{F_{2}}+\frac{L}{\xi} \frac{\dot{F}_{2}}{\sigma_{1}}-\frac{L V_{\mathrm{e} 3}}{\xi \tau F_{2}}+ \\
+\frac{L^{2}\left(V_{\mathrm{e} 3} \dot{F}_{2}-\tau \dot{F}_{2}^{2}\right)+\tau L F_{2} \ddot{F}_{2}}{\xi^{2} \tau F_{2}^{2} \sigma_{1}}-\frac{\nu L \dot{F}_{1}}{\alpha \xi \tau F_{2} \sigma_{1}},
\end{gathered}
$$

where $\sigma_{1}=F_{2}+\frac{L}{\xi \tau}+\frac{L}{\xi} \frac{\dot{F}_{2}}{F_{2}}-\frac{L V_{\mathrm{e} 3}}{\xi \tau F_{2}}$.

Although the traffic Equations (10)-(13) are somewhat complex, the principle of the algebraic methods permits to approximate the measured outputs $\left(\rho_{3}\right.$ and $\left.v_{3}\right)$ by introducing its truncated Taylor polynomial expansion. In this way it is not necessary to design the derivative estimator from a specific dynamic model of the traffic flow. Then, for the generation in time of the measured outputs $\rho_{3}(t)=F_{1}(t)$ and $v_{3}(t)=F_{2}(t)$, consider for example, a 4th order approximation of these smooth signals, $F_{i}(t), i=1$, 2 ,

$$
\frac{d^{4} F_{i}}{d t^{4}}=0
$$

In the operational domain [22] Equation (14) is written as

$$
s^{4} F_{i}(s)-s^{3} F_{i}(s)-s^{2} \dot{F}_{i}(0)-s \ddot{F}_{i}(0)-F_{i}^{(3)}(0)=0 .
$$

The algebraic manipulations as described in [1] and transformation of the obtained expressions in the time domain provide approximations of the first and second order time-derivatives of $F_{i}(t)$, 
(16)

$$
\left\{\begin{array}{l}
{\left[\frac{d F_{i}}{d t}\right]_{\mathrm{e}}=\frac{-24\left(\int_{t_{i}}^{(3)} F_{i}\right)+96\left(\int_{t_{i}}^{(2)}\left(t-t_{j}\right) F_{i}\right)-72\left(\int\left(t-t_{j}\right)^{2} F_{i}\right)+12\left(t-t_{j}\right)^{3} F_{i}}{\left(t-t_{j}\right)^{4}},} \\
{\left[\frac{d^{2} F_{i}}{d t^{2}}\right]_{\mathrm{e}}=\frac{-24\left(\int(2) F_{i}\right)+96\left(\int\left(t-t_{j}\right) F_{i}\right)-36\left(\left(t-t_{j}\right)^{2} F\right)+8\left(t-t_{j}\right)^{3}\left[\dot{F}_{i}\right]_{\mathrm{e}}}{\left(t-t_{j}\right)^{4}}}
\end{array}\right.
$$

Notice, that for the second order time derivative estimate requires the outcome of the evaluation of the first derivative estimate. This is in complete agreement with the announced triangular structure of the generating system of equations, where $t-t_{j}>0$ is the estimation period. (See [8] and [16], for more details and the principle of resetting when the denominator becomes close to 0 .) Using the above formulas allows obtaining the derivative estimations of the traffic density and mean speed.

\section{Traffic flow control}

The traffic flow control represents the only efficient way to overcome the congestion problems. This section shows the efficiency of the state estimation in order to alleviate these phenomena by implementing the isolated ramp metering control. Such control measurement consists of acting on the traffic demand at the on ramp in order to maintain the traffic density in the merge freeway section close to the critical one. The proposed algorithm is based on the joint use of the sliding mode control and differential flatness. From the first term of Equation (4), the control variable $r_{1}$ can be parameterized in term of the flat output $F_{1}=\rho_{3}$ and its time-derivatives up to three:

$$
r_{1}(t)=\theta_{1} F_{1}^{(3)}+\theta_{2} \ddot{F}_{1}+\theta_{3} \dot{F}_{1}+\theta_{4} \ddot{q}_{\text {out }}+\theta_{5} \dot{q}_{\text {out }}+q_{\text {out }}-q_{\text {in }},
$$

where

$$
\begin{gathered}
\theta_{1}=\frac{L^{3}}{\alpha^{2} v_{2} v_{3}}, \theta_{2}=\frac{L^{2}}{\alpha}\left[\frac{1}{v_{3}}-\frac{2-3 \alpha}{v_{3}}\right], \\
\theta_{3}=\frac{L}{\alpha}\left[\frac{v_{3}(1-\alpha)^{2}}{\alpha v_{2}}+2 \alpha-1\right], \theta_{4}=\frac{L^{2}}{\alpha^{2} v_{2} v_{3}}, \theta_{5}=\frac{L}{\alpha}\left[\frac{1}{v_{3}}-\frac{1-2 \alpha}{\alpha v_{2}}\right] .
\end{gathered}
$$

Given a desired reference trajectory $F_{1}{ }^{*}$ (usually, the reference trajectory is taken around the critical density), for the controlled traffic density at the merge segment, a linearized control law achieving an exponential asymptotic tracking of the trajectory is given by the following expression:

$$
r_{1}(t)=\theta_{1} w+\theta_{2} \ddot{F}_{1}+\theta_{3} \dot{F}_{1}+\theta_{4} \ddot{q}_{\text {out }}+\theta_{5} \dot{q}_{\text {out }}+q_{\text {out }}-q_{\text {in }},
$$

where $w=F_{1}^{(3)}$ is an auxiliary control input. Notice that the control variable in that equation needs a derivative estimation of the ouput flow. Such estimation is obtained in the same way as for $F_{1}$ and $F_{2}$. Using the high order sliding mode 
control principle and assuming that the sliding surface $s=F_{1}-F_{1}{ }^{*}$, the auxiliary control input is

$$
w=-\varsigma \operatorname{sign}\left(\dot{s}+\zeta|s|^{\frac{1}{2}} \operatorname{sign}(s)\right)
$$

Substituting (19) in (18) provides the control algorithm for isolated ramp metering.

Simulation results. For the simulation purpose, consider the freeway stretch depicted in Fig. 1 and assume that the two loop-detectors allowing the measurement of the traffic densities in segment 1 and 2 are faulty. Using either the sliding mode observer or the state reconstructor based on numerical differentiation allows the obtaining of the missing data of $\left(\rho_{1}, v_{1}\right)$ and $\left(\rho_{2}, v_{2}\right)$. We have chosen the trapezoidal traffic demand, which is more or less comparable to real-data (Fig. 4).

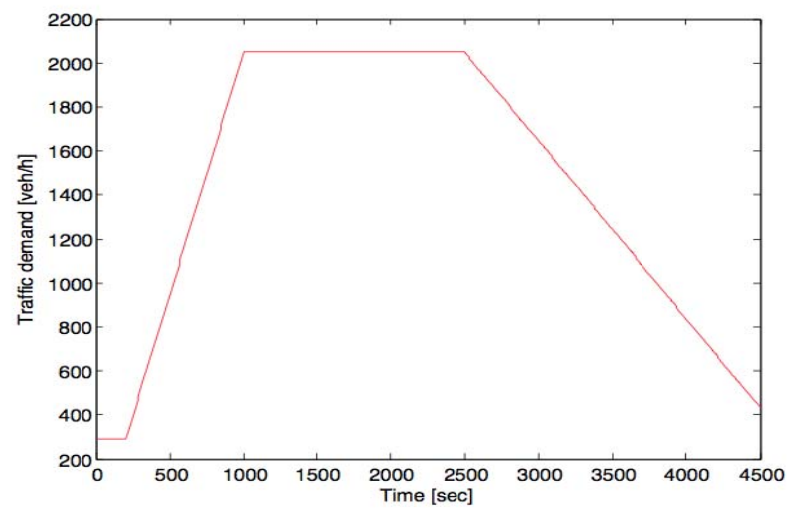

Fig. 4. Example of the used traffic demand

The simulation time is about $1 \mathrm{~h} 25 \mathrm{~min}$. The model parameters are provided in Table 1.

Table 1. Model parameters

\begin{tabular}{|l|c|c|}
\hline \multicolumn{1}{|c|}{ Parameter } & Value & Unit \\
\hline Critical density & 35.86 & $\mathrm{veh} / \mathrm{km}$ per lane \\
\hline Free-flow speed & 105 & $\mathrm{~km} / \mathrm{h}$ \\
\hline Maximal density & 180 & $\mathrm{veh} / \mathrm{km}$ per lane \\
\hline Segment Length & 0.5 & $\mathrm{~km}$ \\
\hline$\alpha$ & 0.77 & - \\
\hline$a$ (May fundamental diagram) & 0.8014 & - \\
\hline$\varsigma$ (Controller parameter) & 0.1 & - \\
\hline$\zeta$ (Controller parameter) & 0.05 & - \\
\hline
\end{tabular}

In the no-control case, Figs $5 \mathrm{a}$ and $\mathrm{b}$ show the densities and the mean speeds evolutions for the measured and estimated state variables. 


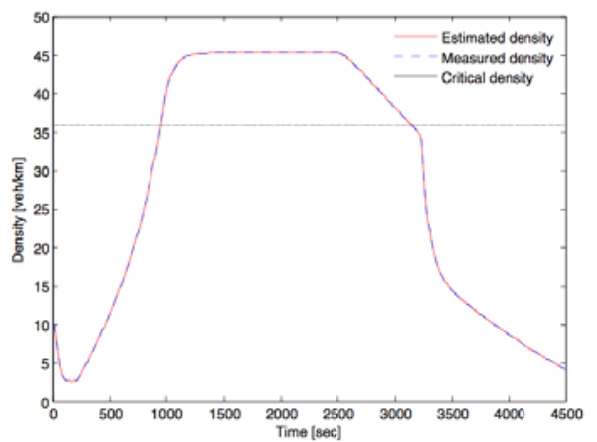

a

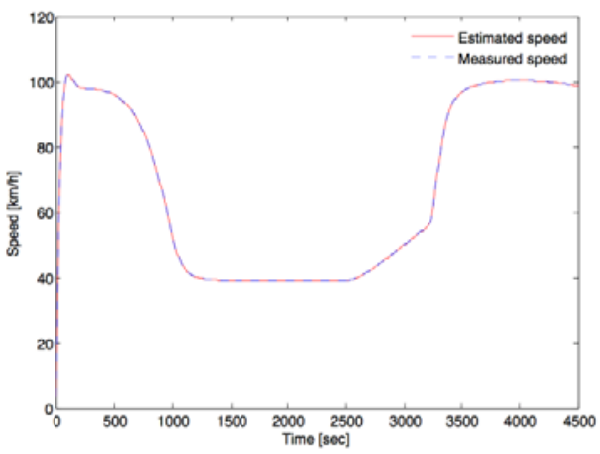

b

Fig. 5. Densities (veh/km) and speed $(\mathrm{km} / \mathrm{h})$ time evolution: no-control case

These figures show that after about 12 minutes, the traffic reaches and exceeds the critical density (Fig. 5a), which means that the freeway stretch is in a congestion mode. This fact is illustrated by the speed evolution, which decreases consequently (Fig. 5b).

Remark that the implementation of our control algorithm shows very encouraging results (Fig. 6), where the densities are maintained around the desired value. This fact is confirmed by the increase in the speed values.

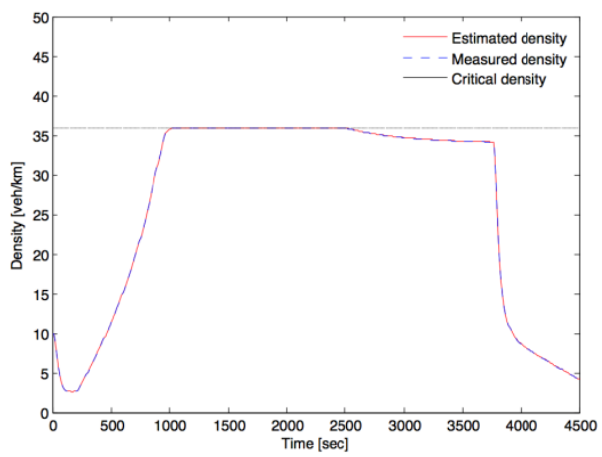

a

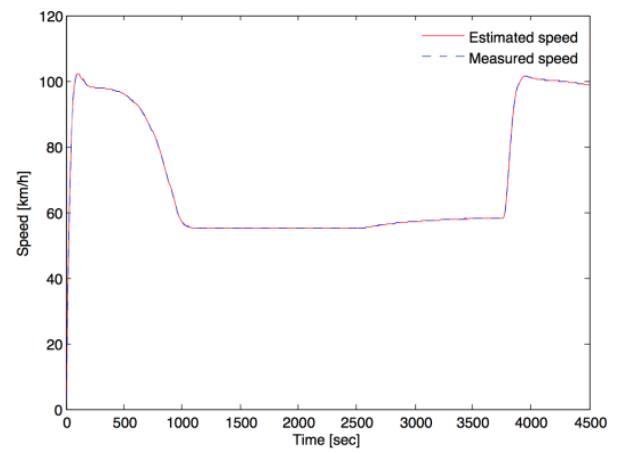

$\mathrm{b}$

Fig. 6. Densities (veh/km) and speed $(\mathrm{km} / \mathrm{h})$ time evolution: control case

Fig. 7 illustrates the control variable evolution, where $0.2 \leq r_{1} \leq 1$.

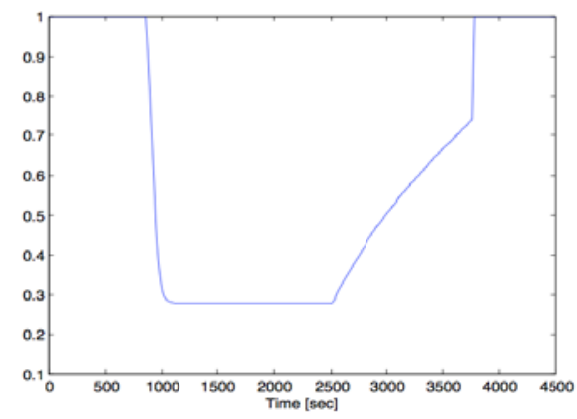

Fig. 7. Control variable evolution 
Obviously, the implementation of the ramp-metering algorithm leads to the queue formation depicted in Fig. 8.

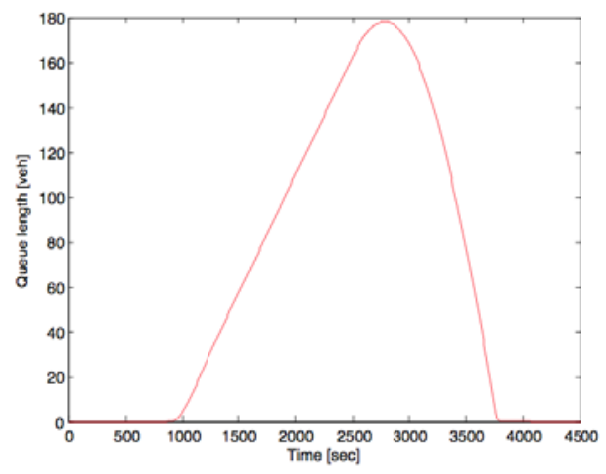

Fig. 8. Queue length time evolution

\section{Conclusion}

The studies presented in this paper provide a new way to deal with traffic flow control in case of missing data or when a loop detector is faulty. Two approaches for state estimation were proposed to tackle this important problem. The first one is based on the so-called super-twisting sliding mode technique. The second one, which provides also robust estimation, is based on the use of newly introduced algebraic methods. Having this needed information, we applied a ramp-metering algorithm, which combines the high order sliding mode control and differentially flat systems concept.

Further on the research will deal with comparative studies of the most widely used techniques in the traffic area, such as Kalman filters and their extensions. This fact leads to the proposal of a coordinated ramp-metering algorithm in the future works, which takes measurements of all the studied freeway networks. In addition, another study is conducted in order to develop integrated traffic flow control combining several traffic flow management actions.

Acknowledgement: This paper is partly supported by FP7 project 316087 ACOMIN: "Advance computing and innovation”.

\section{References}

1. A b o u a ï s s a, H., V. I o r d a n o va. Algebraic Methods for Traffic Flow Densities Estimation. Cybernetics and Information Technologies, Vol. 13, 2013, No 4, pp. 5-17.

2. D i o p, S., M. Fli e s s. On Nonlinear Observability. - In: Proc. of 1st Europ. Control. Conf., Hermès, 1991, pp. 152-157.

3. D i o p, S., M. F l i e s s. Nonlinear Observability, Identifiability and Persistent Trajectorie. - In: Proc. of 26th IEEE Conf. Decision Control, Brighton, 1991, pp. 714-719.

4. Fillipov, A. F. Differential Equations with Discontinuous Right-Hand Side. - American Mathematics Society Transactions, Vol. 62, 1960, pp. 199-231.

5. F l i e s s, M., H. S i r a-R a m ír e z. Reconstructeurs d'état. Comp. Rend. Acad. Sci. Paris, Ser. I, 338, 2004. pp. 91-96. 
6. Fli e s s, M., C. J o i n, H. S ira-Ra mír ez. Robust Residual Generation for Linear Fault Diagnosis: An Algebraic Setting with Examples, Internat. - J. Control, Vol. 77, 2004, pp. 1223-1242.

7. Fli es s, M., C. Jo in, H. Mounier. An Introduction to Nonlinear Fault Diagnosis with an Application to a Congested Internet Router. - In: Lecture Note Advances in Communication and Control Networks. Vol. 308. Springer, 2005.

8. Fli e s s, M., C. J o i n, H. S i ra-R a mír e z. Nonlinear Estimation Is Easy. - Int. J. Model. Identif. Control, 2008, No 4, pp. 12-27.

http://hal.archives-ouvertes.fr/inria-00158855/fr/

9. F l o q u e t, T. Contributions à la commande par modes glissants d'ordre supérieures. PhD Thesis, 2000.

10. G a z i s, D. C., C. H. Kn a p p. On-Line Estimation of Traffic Densities from Times-Series of Flow and Speed Data. - Transportation Science, 1971, No 5, pp. 283-302.

11. Hilliges, M., W. Weidlich. A Phenomenological Model for Dynamic Traffic Flow in Networks. - Transportation Research B., 1995, No 6, pp. 407-431.

12. Kn a p p, C. H. Traffic Estimation and Density Estimation for Single and Multi-Lane Traffic. Transportation Science, 1973, No 7, pp. 75-84.

13. Kohan, R. Robust State Estimation and Control of Highway Traffic Systems. PhD Thesis, Electrical and Computer Engineering, University of Toronto, 2001.

14. Maji d, H., H. A b o u ä s sa, D. J olly, G. Morvan. State Reconstructor for Real-Time Freeway Ramp Metering. - In: Proc. of 10th IEEE International Conference on Networking, Sensing and Control, ICNSC, Paris, France, April, 2013.

15. M a y, A. D. Traffic Flow Fundamentals, Transportation and Traffic Theory in the 21st Century. In: ISTTT. Englewood Cliffs, NJ, Prentice-Hall, 1990.

16. Mboup, M., C. Join, M. Fliess. A Revised Look at Numerical Differentiation with an Application to Nonlinear Feedback Control. - In: 15th Mediterrean Conference on Control and Automation (MED.07), 2007. http://hal.inria.fr/inria/00142588/fr/

17. M i h a y lova, L., R. B o e l. A Particle Filter for Freeway Traffic Estimation. - In: Proc. of 43rd IEEE Conf. on Decision and Control, 14-17 December 2004, Atlantis, Paradise Island, Bahamas, pp. 2106-2111.

18. N a h i, N. E., A. N. Trivedi. Recursive Estimation of Traffic Variables: Section Density and Average Speed. Tech. Report, 1976. University of Southern California, Los Angeles.

19. Rolink, M., T. Boukhobza, D. S a uter. High Order Sliding Mode Observer for Fault Actuator Estimation and Its Application to the Three Tanks Benchmark. In Author manuscript, 2006.

20. S u n, X., L. M u n õ z, R. H o r o w i t z. Mixture Kalman Filter Based Highway Congestion Mode and Vehicle Density Estimator and Its Application. - In: American Control Conference, 2004, pp. 2098-2103.

21. W a n g, Y., M. P a p a g e or g i o u, A. M e s s m e r. Real-Time Freeway Traffic State Estimation Based on Extended Kalman Filter: Adaptive Capabilities and Real Data Testing. - Transport. Research A, 2008, No 42, pp. 1340-1358.

22. Y o s i d a, K. Operational Calculus: A Theory of Hyperfunctions. Springer, 1984 (Translated from Japanese). 